\title{
FUNCTIONAL RESPONSES: A QUESTION OF ALTERNATIVE PREY AND PREDATOR DENSITY
}

\author{
Britta Tschanz, ${ }^{1}$ Louis-FÉlix Bersier, ${ }^{2}$ And Sven Bacher ${ }^{1,3}$ \\ ${ }^{1}$ Zoological Institute, Community Ecology, University of Bern, Baltzerstr. 6, CH-3012 Bern, Switzerland
${ }^{2}$ Unit of Ecology and Evolution, Department of Biology, University of Fribourg, Ch. du Musée 10, CH-1700 Fribourg, Switzerland
}

Abstract. Throughout the study of ecology, there has been a growing realization that indirect effects among species cause complexity in food webs. Understanding and predicting the behavior of ecosystems consequently depends on our ability to identify indirect effects and their mechanisms. The present study experimentally investigates indirect interactions arising between two prey species that share a common predator.

In a natural field experiment, we introduced different densities of mealworms (Tenebrio molitor), an alternative prey, to a previously studied predator-prey system in which paper wasps (Polistes dominulus) preyed on shield beetle larvae (Cassida rubiginosa). We tested if alternative prey affects predation on the first prey (i.e., the predator-dependent functional response of paper wasps) by modifying either interference among predators or the effective number of predators foraging on shield beetles. Presence of mealworms significantly reduced the effective number of predators, whereas predator interference was not affected. In this way, the experimentally introduced alternative prey altered the wasps' functional response and thereby indirectly influenced C. rubiginosa density. In all prey-density combinations offered, paper wasps constantly preferred $T$. molitor. This led to an asymmetrical, indirect interaction between both prey species: an increase in mealworm density significantly relaxed predation on $C$. rubiginosa, whereas an increase in $C$. rubiginosa density intensified predation on mealworms. Such asymmetrical outcomes of a fixed food preference can significantly affect the population dynamics of the species involved. In spite of the repeated finding of a Type III functional response in this system, our experiment did not reveal switching behavior in paper wasps. The variety of mechanisms underlying direct and indirect interactions within our study system exemplifies the importance of incorporating alternative prey when investigating the impact of a generalist predator on a focal prey population under realistic field conditions.

Key words: alternative prey; Cassida rubiginosa; Cirsium arvense; effective predator density; Polistes dominulus; predation; predator-dependent functional response; predator interference; preference; prey switching; ratio dependence; Tenebrio molitor.

\section{INTRODUCTION}

Most predators do not only feed on a single prey but rather exploit diverse prey species and are therefore involved in a complex web of interactions (Strauss 1991). The impact of a generalist predator on any one focal prey species does not only depend on the abundance and susceptibility of that prey (direct effects) but also on the presence and abundance of alternative prey species that share the same enemy (indirect effects: Holt 1977, Abrams 1987, Holt and Lawton 1994). At short time scales, i.e., within a single predator generation, availability of alternative prey was reported to modulate the consumption rate on a focal prey species via the predator's functional response (Murdoch 1973, Holt and Lawton 1994). As an important consequence thereof, generalist predators can contribute to density-

\footnotetext{
Manuscript received 11 September 2006; revised 30 October 2006; accepted 3 November 2006. Corresponding Editor (ad hoc): E. L. Preisser.

${ }^{3}$ Corresponding author.

E-mail: sven.bacher@zos.unibe.ch
}

dependent regulation of prey populations, which potentially expands to long-term effects over multiple predator generations (reviewed in Holt and Lawton 1994). Predictions of the dynamics of complex food web systems therefore critically depend on an accurate understanding of the relative importance of direct and indirect effects in multispecies predator-prey systems.

The most general mechanism underlying short-term effects within a generalist predator-multiple prey system is that capture and consumption of alternative prey reduces the amount of time available for encountering a focal prey (Chesson 1989). If the predator does not prefer either species, indirect effects occurring between two prey species exclusively depend on their abundance: An increased abundance of prey A relaxes predation on prey $\mathrm{B}$; conversely, a decrease in prey A density intensifies predation on prey B (and vice versa). Thus, higher abundances of one of the two prey species are expected to have a short-term positive effect on the other prey. This type of indirect interaction is called apparent mutualism and was explored theoretically by several 
authors (Abrams 1987, Holt and Kotler 1987, Abrams and Matsuda 1993, 1996, Holt and Lawton 1994).

However, if a generalist predator constantly prefers one of the prey species to the other, the outcome of indirect interactions is more difficult to predict (Eubanks and Denno 2000). If the predator prefers prey B, an increase in prey B density will lead to a benefit for prey A because, in this situation, the predator will increasingly exploit its preferred prey. In contrast, an increase in prey $\mathrm{A}$ abundance will not necessarily reduce predation on the preferred prey B. In the case of fixed preference, which is maintained irrespective of the relative availabilities of alternative food types, indirect interactions between prey species may thus show an asymmetrical pattern, suppressing the preferred prey more than the less preferred one (Eubanks and Denno 2000).

A third possible reaction of the predator is prey switching, in which preference is instantaneously adapted when prey availabilities change: Predators concentrate on temporarily abundant prey species, eating them disproportionately more often and disproportionately ignoring rare prey (Murdoch 1969, Murdoch et al. 1975, Murdoch and Oaten 1975). Switching behavior was reported to frequently generate Type III functional responses (Holling 1966) having a sigmoidal relationship to prey abundance (Murdoch and Oaten 1975). Type III functional responses contain a stable equilibrium between prey production and predation and therefore can determine the predator's ability to regulate the density of its prey and to stabilize prey populations in a multiple prey system (Murdoch 1969, Murdoch and Oaten 1975).

However, our present understanding of these mechanisms predominantly relies on theoretical models, and the scant experimental evidence is largely restricted to laboratory studies under simplified conditions that are difficult to apply to natural situations. Establishing empirical examples in different natural systems is methodologically challenging due to the secretiveness of the predation event. However, such data are imperative in order to give a sense of the generality of indirect effects and their potential mechanisms (Wootton 2002). Among other experimental methods, Wootton (2002) proposes to start with a known direct functional relationship between two species and test whether there is any deviation from predicted effects when species composition is changed. Accordingly, we changed species composition in the present study by introducing an alternative prey species to a previously studied predator-prey system (Schenk and Bacher 2002, Schenk et al. 2005, Tschanz et al. 2005; B. Tschanz, L. F. Bersier, and S. Bacher, unpublished manuscript). We investigated if the presence of alternative prey affects predation on the first prey, specifically the predator's functional response. The important role of the functional response in determining indirect effects within food webs was underlined by several authors (Berlow et al. 1999, Abrams and Ginzburg 2000, Abrams 2001).
They reasoned that a predator's functional response represents one of the most important components of the relationship between per capita growth rates and population densities in natural environments (Abrams 2001). Understanding the functioning of this relationship may be the key to estimating the strength of interspecific interactions and to filling the gap between theory and experiments in community ecology (Laska and Wootton 1998, Berlow et al. 1999).

Paper wasps Polistes dominulus (Hymenoptera, Vespidae) are abundant generalist predators in South and Central Europe (Gauld and Bolton 1988, Witt 1998). By means of continuous video surveillance, previous investigations reported paper wasps to feed on Cassida rubiginosa larvae (Coleoptera, Chrysomelidae) in the field. In these previous experiments, we manipulated prey density (Schenk and Bacher 2002), prey and predator density (Schenk et al. 2005), and prey vulnerability (B. Tschanz, L. F. Bersier, and S. Bacher, unpublished manuscript) in this system and identified the functional response of $P$. dominulus as predator-dependent Type III, taking an intermediate form between prey- and ratio-dependence (Schenk et al. 2005; B. Tschanz, L. F. Bersier, and S. Bacher, unpublished manuscript).

Estimating the impact of a generalist predator on a focal prey population generally requires offering the possibility of a choice among multiple prey types (Abrams and Ginzburg 2000). In most field cage and laboratory studies, however, single-prey species functional response experiments are used in order to investigate predator behavior. This can lead to misinterpretation, e.g., to an overestimation of real-world effects (Eubanks and Denno 2000). Conversely, the aforementioned unrestricted paper wasp-shield beetle experiments allowed predators to freely move in the surrounding of the experimental patch and therefore to forage on prey other than C. rubiginosa. The occurrence of alternative prey species as well as their densities may have influenced shield beetle predation. In order to specifically measure possible indirect effects between different prey species, we experimentally offered, in this study, mealworms (Coleoptera, Tenebrionidae, Tenebrio molitor L.) as alternative prey and tested whether their density affected predation on $C$. rubiginosa under natural field conditions. Note that mealworms are not natural prey of paper wasps and are used here as surrogate for alternative prey in general.

The nature of mechanisms underlying direct interactions between $P$. dominulus and their prey on the one hand and indirect interactions between $C$. rubiginosa and other prey species on the other hand is determined by the predator's food preference and its functional response. We therefore investigated the paper wasps' food preference and density dependence (fixed preference or prey switching) and integrated alternative prey density in the functional response analysis in two different ways. As a consequence, we were able to 
estimate the importance of alternative prey in modifying either intraspecific predator interference or the effective number of predators foraging for shield beetles and thereby shaping the predator-dependent functional response of $P$. dominulus.

\section{Methods \\ Experimental site and set-up}

The experiment was carried out in the garden of the Zoological Institute (University of Bern, Bern, Switzerland) on a patch of $30 \mathrm{~m}^{2}$, where the naturally growing vegetation predominantly consisted of Solidago canadensis L. and creeping thistle Cirsium arvense (L.). The patch was located in the margin of the Institute's courtyard, belonging to a larger, partly uncultivated meadow with a mixture of flowering plants and surrounded by orchard trees and hedges. The same site had been used in earlier studies on paper wasp predation on shield beetle larvae (Schenk and Bacher 2002, Bacher and Luder 2005, Schenk et al. 2005, Tschanz et al. 2005; B. Tschanz, L. F. Bersier, and S. Bacher, unpublished manuscript). We used last (i.e., fifth) instar larvae of shield beetles and last instar larvae of mealworms in our experiments. Shield beetle larvae and mealworms differ in their size and appearance: shield beetle larvae are smaller (length $\sim 0.8 \mathrm{~cm}$ ), soft-bodied, oval shaped with spines at their circumference, and carry a shield made out of their own feces on their back (Bacher and Luder 2005), while mealworms are larger (length $\sim 1.5 \mathrm{~cm}$ ), protected by a hard exoskeleton, and have the general appearance of caterpillars.

Sixty thistle shoots of similar height and stature were marked individually and assigned as either "hidden" (i.e., shoots were concealed by dense surrounding vegetation, $N=30$ ) or "exposed" (i.e., free-standing shoots without contact to neighboring vegetation, $N=$ 30) (Tschanz et al. 2005).

Combinations of different prey densities $(C$. rubiginosa: $N=0,10,20,30$ larvae; T. molitor: $N=0,10,20,30$ larvae) were investigated in a factorial design. Except $0 / 0$, all $C$. rubiginosa $/ T$. molitor density combinations were assigned randomly to the experimental days, such that at the end of the experiment each combination had been investigated twice (30 experimental days). Early in the morning (09:00) on every experimental day, i.e., before wasps started foraging (Schenk and Bacher 2002), the entire patch was searched for naturally occurring shield beetle larvae, which were then removed prior to the treatment application. Subsequently, the assigned numbers of shield beetle larvae and mealworms were allocated to the same number of randomly selected thistles such that each selected thistle received one prey individual. For each prey species, half of the larvae were placed on "exposed" and the other half on "hidden" thistles. Preceding experiments established that $C$. rubiginosa larvae do not leave the host plant they are placed on and that mortality from causes other than predation is practically nonexistent in late larval instars
(S. Bacher, unpublished data). Thus, all larvae having vanished during the experiment could be attributed to predation. Since mealworms do not naturally occur on $C$. arvense and easily fall off the plants, they had to be fixed to the leaves by means of small fishhooks (size 9). This way the mealworms stayed alive, but were unable to change their location.

Predation was investigated by continuous video surveillance of individual prey (five cameras focusing on $C$. rubiginosa larvae and five cameras focusing on $T$. molitor larvae; except in the cases of only one prey species being offered, where all 10 cameras were monitoring individuals of that prey species), as described in detail elsewhere (Schenk and Bacher 2002). We knew from previous experiments that the almost exclusive predators of shield beetle larvae at our site were paper wasps $P$. dominulus freely foraging in the Institute's garden and its surroundings. Paper wasps are polyphagous, feeding on a variety of arthropod prey species. There are no known predators of paper wasps at our site. The nests of the paper wasps were located in the courtyard of the Institute's garden; predators were thus approaching our experimental site from only one direction. This allowed us to measure each experimental plant's distance to the patch margin from where the wasps approached (accuracy $1 \mathrm{~m}$ ).

Three times a day, the number of predators foraging within the experimental patch was determined for 30 minutes ("sampling periods": 10:30-11:00, 13:30-14:00, 16:30-17:00). All predators observed were caught by sweep netting, marked individually with acrylic color, and released afterwards. At the end of each sampling period (at 11:00, 14:00, and 17:00) and early the next morning, all experimental plants were checked for remaining larvae in order to document the time course of prey depletion. No experiments were conducted on rainy days. Predation recording took place between 15 June and 28 July 2005.

\section{Analyses}

For each observation period (09:00-11:00, 11:0014:00, and 14:00-17:00), predation rates of C. rubiginosa and $T$. molitor were calculated as the number of prey eaten $(\Delta N=$ the number of $C$. rubiginosa larvae eaten and $\Delta A=$ the number of $T$. molitor larvae eaten) divided by the corresponding prey density at the beginning of each period $(N=C$. rubiginosa density and $A=T$. molitor density). Daily predation rates and total predation rates per plant, summed over the entire duration of the experiment, were compared between prey species by paired $t$ tests.

We modeled the probability of a prey individual being eaten by a predator with a generalized linear mixedeffects model (GLMM; function glmmPQL in the statistical software $\mathrm{R}$, version 2.2.0; available online $)^{4}$

\footnotetext{
${ }^{4}\langle$ http://www.r-project.org $\rangle$
} 
with a logit link function and a binomial error distribution. The experimental day and the experimental plant were used as random variables, and the distance of plants to the margin of the experimental patch (in meters), the exposure of the plants ("hidden" or "exposed"), the number of wasps foraging during the experimental period (see Eq. 2), the number of $C$. rubiginosa larvae, and the number of $T$. molitor larvae offered at the beginning of the experiment as fixed effects. The analysis was separately performed on data for $C$. rubiginosa and $T$. molitor (each $N=480$ ). In this analysis, we only used data from the first observation period (09:00-11:00), where predation rates were moderate. Including data from later observation periods, where the proportion of prey eaten was close to 1 , may obscure the effect of the factors investigated because predators increasingly include non-preferred prey in their diet as preferred prey becomes rarer.

As described by Murdoch et al. (1975), the predator's food preference was measured by estimating the parameter $c$ in the following equation:

$$
P_{1, i j}=c F_{1, i j} /\left(1-F_{1, i j}+c F_{1, i j}\right) .
$$

$F_{1}$ indicates the proportion of prey species 1 offered, $P_{1}$ describes its proportion in the diet for each experimental day $i$ and each observation period $j$. We conducted a nonlinear regression to estimate $c$, using the sequential quadratic algorithm of SPSS (version 13.0) and a bootstrap procedure, the latter providing the parameter's standard error and confidence intervals. A value of $c<1$ indicates preference for the focal prey species 1; the predator does not exhibit any preference if 1 is included in the confidence interval of $c$.

Additionally, simple preference indices $c_{i j}$ were calculated for each experimental day $i$ and each observation period $j$ by dividing the proportion of $T$. molitor to $C$. rubiginosa eaten $(\Delta A / \Delta N)$ by the proportion of their abundances $(A / N$; Murdoch et al. 1975). We tested by multiple regression analysis if the wasps' preference indices depended on the total number of prey available and on the total number of predators.

The wasp individuals caught by sweep netting were used to estimate the total number of wasps $(P)$ foraging at the site within each observation period of a given day (d). Based on the cumulative number of predation events per hour originating from previous studies (Schenk and Bacher 2002, Schenk et al. 2005; B. Tschanz, L. F. Bersier, and S. Bacher, unpublished manuscript), we had an estimation of the wasps' activity in the course of a day (B. Tschanz, L. F. Bersier, and S. Bacher, unpublished manuscript). Fitting these cumulative data with a $\gamma$-distribution, we were able to compute the proportion of wasps $\left(p_{h}\right)$ expected to be found during a given 30 -min period $(h)$ with respect to the sampling intervals $(h=1-16$, with $h=4,10$, and 16 the sampling intervals corresponding to the periods of catching, i.e., 10:30-11:00, 13:30-14:00 and 16:3017:00). Knowing the number of wasps caught between
10:30-11:00 (w) and the average number of wasps caught within the first sampling periods over all experimental days $(M)$, the expected total number of predators for the first observation period (09:00-11:00) on day $(d)$ was calculated as

$$
P_{d}=\sum_{h=1}^{3} p_{h} M+w .
$$

Using the corresponding values for $M$ and $w$, respectively, of the second (and third) sampling period and summing the expressions $p_{h} M$ from $h=5-9$ (and from $h=11-15$ ) revealed the total number of predators between 11:00 and 14:00 (and between 14:00 and 17:00).

As similarly described elsewhere (Schenk et al. 2005; Tschanz et al. unpublished manuscript), we modified the method of Arditi and Saïah (1992) to calculate Type III functional responses, explicitly allowing for prey depletion during the experiment. Without alternative prey, the actual number of $C$. rubiginosa eaten is given by

$$
\begin{aligned}
\Delta N= & {\left[P^{2 m}+b N^{2} t_{h}+b N P T\right.} \\
& \left. \pm \sqrt{-4 b^{2} N^{3} P t_{h} T+\left(P^{2 m}+b N^{2} t_{h}+b N P T\right)^{2}}\right] \\
& \div 2 b N t_{h} .
\end{aligned}
$$

$N$ represents the number of shield beetles at the start of each observation period, $P$ the total number of wasps present at the experimental site during each observation period (estimated by Eq. 2 or its adaptation to the second or third observation period), and $T$ the foraging time of wasps, i.e., four or six 30-min periods, respectively (corresponding to the duration of the three observation periods). The parameter $m$ is a measure of predator interference, which takes the value 0 for pure prey-dependence and 1 for pure ratio-dependence. It was estimated by nonlinear regression, as noted later in Methods. According to the results of an earlier experiment at the same site (see Schenk and Bacher 2002: Table 3), we set the "search efficiency" as $b=0.004$ and the handling time as $t_{h}=0.17$. By doing this, we assume that these parameters are characteristic of this system at this site. We also performed a sensitivity analysis where $b$ and $t_{h}$ were varied in a plausible range which did not affect our conclusions (see Results).

The effect of alternative prey density $(A)$ on the predatordependent functional response toward $C$. rubiginosa can be tested by two different approaches. The presence of alternative prey can either reduce the effective number of predators foraging for $C$. rubiginosa $(P)$ or the magnitude of interference between predators $(m)$ or both. In the first case, predator individuals concentrating on alternative prey cease to be relevant foragers of $C$. rubiginosa larvae. The predator density important for C. rubiginosa must thus be adjusted by discounting the number of wasps foraging for mealworms from the total predator density. With increasing densities of alternative prey, a higher number of 


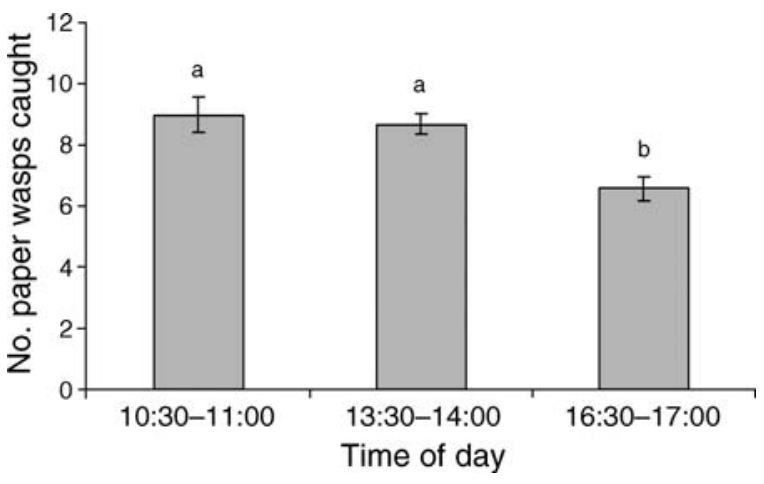

FIG. 1. Number of Polistes dominulus wasps (mean \pm SE) foraging at the experimental site and caught by sweep-netting within the three sampling periods. Different letters indicate significant differences (post hoc tests, Tukey's LSD, $P<0.001$; $N=30 \mathrm{~d}$ ).

predators is expected to exclusively forage for alternative prey. Consequently, predator densities relevant for $C$. rubiginosa should tend toward 0 at infinitely high densities of alternative prey. On the other hand, if no alternative prey is offered at all, predators will exclusively forage for shield beetles, and the expression for predator density should not change. For parsimony, we modeled this mechanism by assuming a simple hyperbolic relationship and replaced $P$ with $P /(q A+1)$ in Eq. 3 . The parameter $q$ can be interpreted as a measure of the magnitude of the effect of alternative prey density on the effective number of predators relevant for shield beetles. If alternative prey actually distracts predators from foraging for $C$. rubiginosa, $q$ should be greater than 0 , i.e., the confidence interval of $q$ should not include 0 .

Alternatively, we can assume that the presence of alternative prey reduces intraspecific predator interference $(m)$. At an increased density of alternative prey, predators are expected to increasingly feed on alternative prey. Shield beetle depletion should thus not occur as quickly as without alternative prey. Consequently, "easy to find" prey will remain longer in the system (Tschanz et al. 2005; B. Tschanz, L. F. Bersier, and S. Bacher, unpublished manuscript). Indirect predator interference caused by heterogeneous prey vulnerability should therefore decrease and, at infinite high densities of alternative prey, even converge to 0 . However, in the absence of alternative prey, the strength of predator interference should not change. Again, this scenario can be modeled by replacing $m$ with $m /(x A+1)$ in Eq. 3 . Similarly to $q$, the parameter $x$ can be taken as a measure of the magnitude of the effect of alternative prey density on indirect predator interference. The parameters $q, x$, and $m$ were simultaneously estimated by nonlinear regression of data from all three observation periods using the sequential quadratic algorithm of SPSS (version 13.0) and a bootstrap procedure, which provided the parameters' standard errors and confidence intervals.

\section{Results}

\section{Predators and predation rates}

We videotaped a total of 271 prey individuals (146 mealworms and 125 shield beetle larvae) and 264 predation events. In front of the cameras, all mealworms were eaten, $94.5 \%$ by $P$. dominulus and $5.5 \%$ by yellow jackets, Vespula sp. In C. rubiginosa, paper wasps were responsible for $99.2 \%$ of all predation events. One individual disappeared during the night, and seven shield beetle larvae remained undetected by predators. Thus, patterns in predation can be attributed almost exclusively to the paper wasp $P$. dominulus.

The number of wasps foraging at the experimental site did not depend on the number of prey offered (linear regression: all $P>0.05$ for each prey species and sampling period). The numbers of wasps caught were significantly higher in the first two sampling periods $(09: 00-11: 00,11: 00-14: 00)$ than in the third one (14:0017:00; one-way ANOVA, post hoc tests [Tukey's LSD]: $P<0.001$; Fig. 1). This was expected due to the reduced activity of wasps and the reduced number of prey available in the afternoon (see Schenk and Bacher 2002; B. Tschanz, L. F. Bersier, and S. Bacher, unpublished manuscript). Compared to previous studies conducted at the same site (Schenk et al. 2005: Fig. 1; B. Tschanz, L. F. Bersier, and S. Bacher, unpublished manuscript), we recorded considerably higher predator numbers in this study. As a potential consequence thereof, we generally measured high predation rates, especially in the second and third observation period (C. rubiginosa: $35.6 \%$, $72.4 \%$, and $61.6 \%$; T. molitor: $64.4 \%, 90.2 \%$, and $97.8 \%$ ). Within each observation period, mealworms suffered from significantly higher predation rates than shield beetles (paired $t$ tests: $P<0.001, P=0.015, P=0.042$ ). Analyzing total predation rates on individual host plants over all experimental days showed similar results.

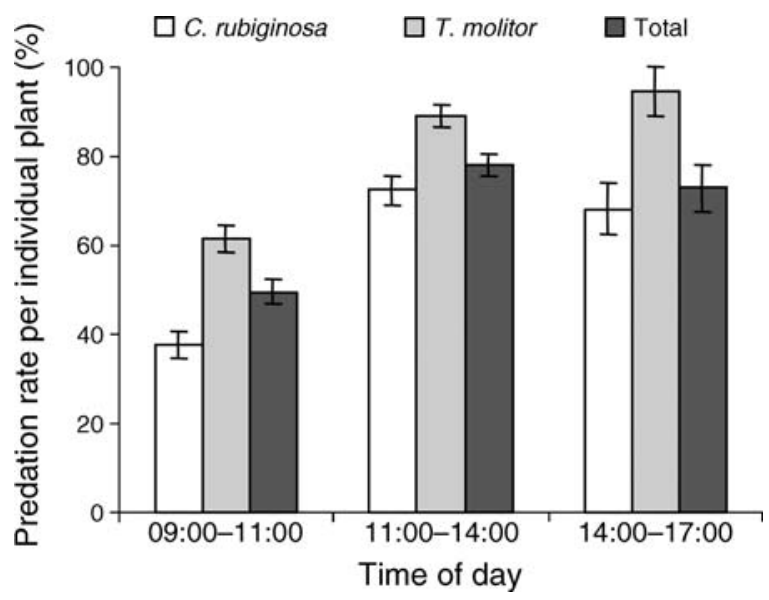

FIG. 2. Total predation rates (percentage of prey eaten by paper wasps [Polistes dominulus]) per individual experimental plant (mean $\pm \mathrm{SE}$ ), shown for every observation period, for each prey species (Tenebrio molitor [mealworms], Cassida rubiginosa [shield beetle larvae]) and their total. 


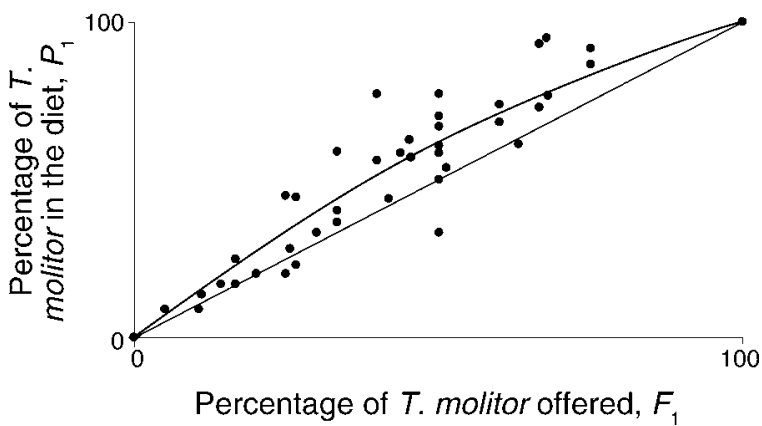

FIG. 3. Polistes dominulus shows a constant preference for mealworms, Tenebrio molitor (nonlinear regression analysis: $c=$ $1.54 ; N=78) . F_{1}$ indicates the proportion of prey species 1 offered, and $P_{1}$ describes its proportion in the diet for each experimental day; $c$ is the preference index. The straight line represents a theoretical situation without food preference $(c=$ $1)$.

Within every observation period, mealworms had higher predation rates than beetle larvae (paired $t$ tests: $P<$ $0.001, P<0.001, P=0.030$; Fig. 2).

During the whole experimental period, we caught 212 individually marked wasps, of which 81 were caught only once, 51 were caught twice, and 31 three times; the remaining 49 wasps were caught more than three times. We also tested if predation rates increased during the experiment but found no indication of this for any prey species during any observation period (all $P>>0.05$ ). Thus, predator learning (of preferred prey) during the experiment could be excluded.

\section{Preference}

In investigating the predator's preference for $T$. molitor, nonlinear regression yielded $c=1.54$ (data from all three observation periods; $N=78, R_{\text {adj }}^{2}=0.96,95 \%$ confidence interval 1.19-1.89; Fig. 3). Since $c=1$ was not included in the parameter's confidence interval, this result indicates a significant preference for mealworms. However, this preference showed no density dependence, i.e., preference indices $c_{i j}$ depended neither on the total number of prey available $(P=0.410)$ nor on the total number of predators $(P=0.381)$. Thus, changing predator-prey ratios did not result in a prey switch in $P$. dominulus prey preference from mealworms to shield beetle larvae.

\section{Correlates of predation}

Higher numbers of predators at the experimental site (calculated as in Eq. 2) caused an increased predation risk for both prey species (GLMM analyses were done on data from the first observation period only; both $P<$ 0.001). The density of conspecifics did not affect predation on individual C. rubiginosa (GLMM: $P=$ 0.126). However, higher mealworm abundances reduced the probability of being eaten for each mealworm individual (GLMM: $P<0.001$ ). Plant distance to patch margin from where the wasps approached was a decisive factor determining the likelihood of predation on both prey species (GLMM: both $P<0.001$ ); prey on host plants close to the margin were more likely to be eaten than prey on plants further away (Fig. 4). In contrast to previous experiments (Tschanz et al. 2005; B. Tschanz, L. F. Bersier, and S. Bacher, unpublished manuscript), we found no effect of host plant exposure on predation of C. rubiginosa (GLMM: $P=0.476$ ). Surprisingly, mealworms on "hidden" plants suffered from marginally higher predation rates than those of "exposed" thistles (GLMM: $P=0.013$ ). The distance to the patch margin was not different for exposed and hidden experimental plants ( $t$ test: $P=0.222$ ).

The common assumption of the two different approaches to integrate alternative prey density in the functional response analysis, namely that predation on C. rubiginosa should be reduced at higher densities of alternative prey, was confirmed by the GLMM $(P<$ 0.001; Fig. 5). In contrast, from the perspective of $T$. molitor, predation on mealworms was significantly intensified at higher shield beetle abundances (GLMM: $P=0.007)$.

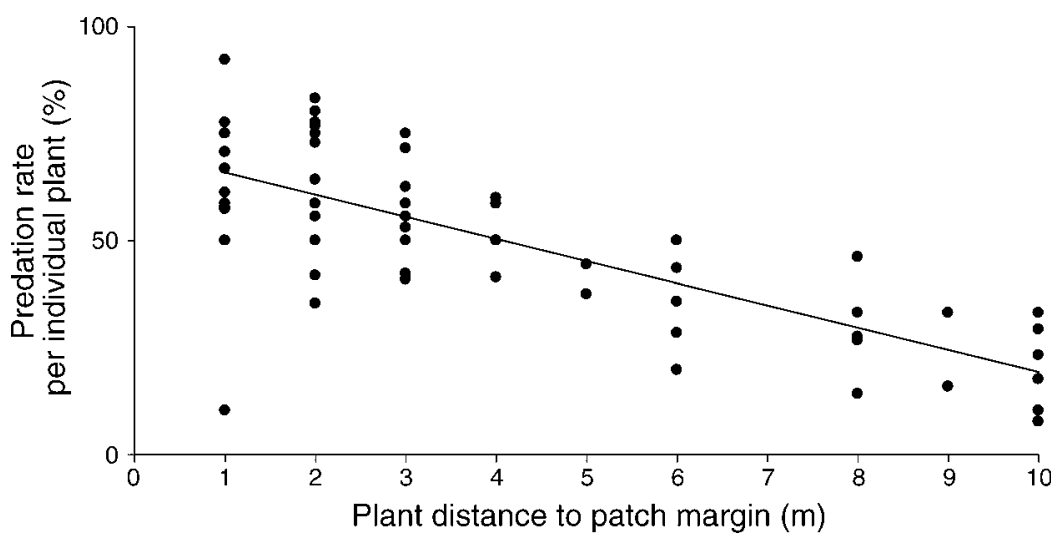

FIG. 4. Impact of plant distance to the patch margin on total predation rates occurring on individual plants (linear regression: $y$ $=-0.052 x+0.711, P<0.001)$. Data points originate from the first observation period $(N=60)$. 


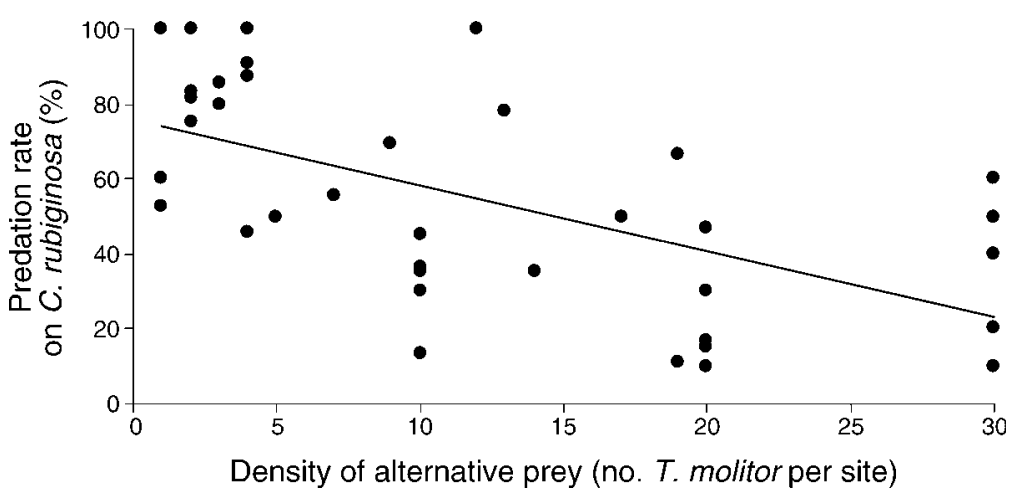

FIG. 5. Predation rates on shield beetle larvae (Cassida rubiginosa), dependent on alternative prey density (linear regression: $y=$ $-0.018 x+0.758, P<0.001 ; N=41$ ). Each data point represents the predation rate on $C$. rubiginosa larvae averaged over one observation period over the entire site. Data points with either no $C$. rubiginosa or no alternative prey were excluded.

\section{Effect on functional response}

Parameter estimation, modeling the effect of alternative prey on the effective predator density using data from all three observation periods $(N=57)$, revealed $q=$ $0.448(N=68, \mathrm{SE}=0.148,95 \%$ CI, $0.152 \leq q \leq 0.745)$. Since $q$ was significantly different from zero, the presence of alternative prey appeared to have affected the predator density relevant for $C$. rubiginosa. The parameter $x$ (modeling the effect of the density of alternative prey on predator interference) was not significantly different from $0(x=0.056, N=68, \mathrm{SE}=$ 0.064 , 95\% confidence interval $-0.071 \leq x \leq 0.183)$. Estimating the magnitude of predator interference revealed $m=0.321(N=68, \mathrm{SE}=0.031,95 \% \mathrm{CI}, 0.258$ $\leq q \leq 0.383$ ). This value was similar to those found in previous studies and confirmed that the functional response took an intermediate form between pure preydependence and pure ratio-dependence (Schenk et al. 2005; B. Tschanz, L. F. Bersier, and S. Bacher, unpublished manuscript). The general pattern for estimates of $q, x$, and $m$ was robust to the use of different plausible values of $b(0.0038 \leq b \leq 0.0042)$ and $t_{h}(0.01$ $\leq t_{h} \leq 0.19$ ).

\section{Discussion \\ Preference}

In all prey density combinations offered, paper wasps preferentially attacked $T$. molitor larvae. Such a fixed food preference of a generalist predator can significantly determine the nature of indirect effects occurring within a multiple prey system (Eubanks and Denno 2000). The paper wasps' preference for one of the two prey types caused an asymmetrical indirect interaction between alternative prey species. An increase in mealworm density significantly relaxed predation on $C$. rubiginosa (Fig. 5), whereas an increase in $C$. rubiginosa density intensified predation on mealworms. At higher mealworm densities, predators thus increasingly exploited their preferred prey, whereas shield beetles indirectly benefited from increased mealworm abundance. By contrast, T. molitor suffered from increased shield beetle abundance. We found no evidence that increased densities of shield beetles attracted more predators, indicating that predators became more efficient in foraging on mealworms at higher densities of the less preferred shield beetles.

When prolonged over long time scales, such asymmetrical outcomes of a fixed food preference may significantly determine the population dynamics of the prey species involved, i.e., their coexistence or exclusion (Holt and Lawton 1994). Predicting the development of specific populations, however, requires knowledge of the predator's behavioral adaptiveness (e.g., how fixed is the predator's preference outside the conditions tested?) and of food web effects (e.g., higher order predation; Holt and Lawton 1994, Kondoh 2003).

Prey switching constitutes a variable, i.e., densitydependent, type of food preference and was reported to frequently generate Type III functional responses (Murdoch and Oaten 1975). In spite of the repeated finding of a Type III functional response in this paperwasp-shield beetle system (Schenk and Bacher 2002, Schenk et al. 2005; B. Tschanz, L. F. Bersier, and S. Bacher, unpublished manuscript), our experiment did not reveal switching behavior in paper wasps. Different reasons may be responsible for our results. First, the specific form of the wasps' functional response may have been created by other mechanisms than prey switching. Learning processes, for example, can cause a more than linear increase in predation rate with higher food availability, which is characteristic for Type III functional responses (Real 1977). An alternative explanation could be that switching may indeed occur in our system but at other prey densities than those studied, as suggested by Mattila and Bonsdorff (1998). However, the realistic and broad range of prey densities tested in our investigation argues against this explanation. Furthermore, other prey species available may have affected the prey choice of paper wasps. Since predators were allowed to freely move in the surroundings of our experimental patch, they could have exhibited switching 
behavior between $C$. rubiginosa and another, unobserved prey species.

\section{Importance of host plant characteristics}

In contrast to previous findings (Tschanz et al. 2005; B. Tschanz, L. F. Bersier, and S. Bacher, unpublished manuscript), prey on "exposed" host plants did not suffer from a higher predation than prey on "hidden" plants in the present study. This was surprising, as many other studies showed that habitat complexity reduces predation rates (e.g., Buckel and Stoner 2000, Finke and Denno 2002, Hoddle 2003). The increased number of predators (compared to our previous studies; Schenk and Bacher 2002, Schenk et al. 2005; B. Tschanz, L. F. Bersier, and S. Bacher, unpublished manuscript) may have forced wasps to also hunt for prey on the more difficult to search "hidden" plants. These results show that the influence of environmental factors can vary between years.

Predation was significantly determined by the host plant's distance to the patch margin: Predators primarily foraged close to the margin (in the outer part of the experimental patch) before they expanded predation to "distant" host plants. Similar to the effect of various host plant exposures (as described in Tschanz et al. 2005), this directed predation from "close" to "distant" plants created heterogeneity in prey vulnerability, which likely contributed to the observed level of predator dependence found in the functional response (B. Tschanz, L. F. Bersier, and S. Bacher, unpublished manuscript).

\section{Density of predators and conspecifics}

Video recordings clearly identified $P$. dominulus as the predominant predator of both prey species. In accordance with this finding, the probability of being eaten significantly increased for both prey at higher numbers of paper wasps present. Offering an increased T. molitor density reduced the probability of being eaten for each mealworm individual (i.e., the risk of predation was "shared" among multiple individuals). In contrast, density of conspecifics did not significantly affect the risk of predation for shield beetle larvae. Higher $C$. rubiginosa densities did thus not result in a decreased (i.e., "shared") predation risk for this prey. This finding represents an indication of a Type III functional response, where predators become increasingly efficient at higher prey abundance (Real 1977).

\section{Parameter estimation, effect of alternative prey on the functional response}

Our finding of an intermediate form of the paper wasps' functional response is in accordance with previous studies (Schenk et al. 2005; B. Tschanz, L. F. Bersier, and S. Bacher, unpublished manuscript). It corroborates the predictions of Abrams and Ginzburg (2000) that both precise prey dependence and ratio dependence should be rare in nature and that functional responses are likely to be affected by species other than prey. Presence of alternative prey significantly reduced the effective number of predators, whereas predator interference was not affected. To our knowledge, our study provides the first experimental evidence that alternative prey can alter the effective predator density. The reasoning behind our mathematical approach was built on the assumption that predator individuals concentrating on mealworms cease to be relevant foragers of $C$. rubiginosa larvae. In our experiment, at higher densities of (preferred) alternative prey, more and more predators fed on mealworms. Indeed, these wasps had to be discounted from the total predator density in the functional response analysis for predation risk on $C$. rubiginosa. Conversely, the magnitude of predator interference did not change with mealworm density. This finding shows that the process of shield beetle depletion in a heterogeneous environment (i.e., indirect predator interference) was not influenced by alternative prey. Similarly, direct predator interference, such as aggression between competing wasps, appeared not to be affected by alternative prey.

Experimentally introducing an alternative prey species to a previously studied predator-prey system significantly affected the known direct functional relationship between $P$. dominulus and $C$. rubiginosa, i.e., the predator's functional response. By modifying the wasps' consumption of shield beetles, alternative prey thus indirectly affected C. rubiginosa densities (Abrams 1987, Holt and Lawton 1994). Our study investigated an example of direct and indirect interactions occurring within a natural generalist predator-multiple prey system and identified the mechanisms underlying them. The variety of such mechanisms, determined by the predator's food preference and modifying the predators' functional response, highlights the importance of incorporating combinations of alternative prey when studying the impact of a generalist on a focal prey population. Using results of isolated, single prey species experiments for modeling complex food webs can lead to misinterpretation and erroneous conclusions about the structure and dynamics of natural predator-prey systems (e.g., Wootton 1994, Müller and Godfray 1999, Eubanks and Denno 2000, Yodzis 2000): Due to the presence of alternative prey within food webs, each predator's functional response may undergo changes instead of having an immutable form. This aspect of indirect interactions between species must ultimately be taken into consideration in models of realistic communities. Investigating indirect effects and their mechanisms in natural systems increasingly gains in importance. Its challenge predominantly lies in its application in many ecological problems, such as biological control, wildlife disease epidemiology, environmental change, and species conservation (Holt and Lawton 1994, Wootton 2002).

\section{ACKNOWLedgments}

We thank Peter Abegglen, Rolf Brechbühl, Wiebke Entling, Markus Fluri, Patrick Jäger, Markus Tschanz, Elva TschanzShigler, Heiri Wandeler, and Corinne Zurbrügg for help in field 
work, and Daniel Engimann and Eduard Jutzi for assistance in technical matters. The paper was greatly improved by comments from Martin Thiel and an anonymous reviewer. This study was funded by the National Centre of Competence in Research (NCCR) Plant Survival, research program of the Swiss National Science Foundation.

\section{Literature Cited}

Abrams, P. A. 1987. Indirect interactions between species that share a predator: varieties of indirect effects. Pages 38-54 in W. C. Kerfoot and A. Sih, editors. Predation, direct and indirect impacts on aquatic communities. University Press of New England, Hanover, New Hampshire, USA.

Abrams, P. A. 2001. Describing and quantifying interspecific interactions: a commentary on recent approaches. Oikos 94: 209-218.

Abrams, P. A., and L. R. Ginzburg. 2000. The nature of predation: Prey dependent, ratio dependent or neither? Trends in Ecology and Evolution 15:337-341.

Abrams, P., and H. Matsuda. 1993. Effects of adaptive predatory and anti-predator behaviour in a two-prey-onepredator system. Evolutionary Ecology 7:312-326.

Abrams, P., and H. Matsuda. 1996. Positive indirect effects between prey species that share predators. Ecology 77:610616.

Arditi, R., and H. Saïah. 1992. Empirical evidence of the role of heterogeneity in ratio-dependent consumption. Ecology 73: 1544-1551.

Bacher, S., and S. Luder. 2005. Picky predators and the function of the faecal shield of a cassidine larva. Functional Ecology 19:263-272.

Berlow, E. L., S. A. Navarrete, C. J. Briggs, M. E. Power, and B. A. Menge. 1999. Quantifying variation in the strengths of species interactions. Ecology 80:2206-2224.

Buckel, J. A., and A. W. Stoner. 2000. Functional response and switching behavior of young-of-the-year piscivorous bluefish. Journal of Experimental Marine Biology and Ecology 245: 25-41.

Chesson, J. 1989. The effect of alternative prey on the functional response of Notonecta hoffmani. Ecology 70: 1227-1235.

Eubanks, M. D., and R. F. Denno. 2000. Health food versus fast food: the effects of prey quality and mobility on prey selection by a generalist predator and indirect interactions among prey species. Ecological Entomology 25:140-146.

Finke, D. L., and R. F. Denno. 2002. Intraguild predation diminished in complex-structured vegetation: implications for prey suppression. Ecology 83:643-652.

Gauld, I., and B. Bolton. 1988. The aculeate apocritans. Pages 218-262 in I. Gauld and B. Bolton, editors. The Hymenoptera. British Museum (Natural History), Oxford University Press, London, UK.

Hoddle, M. S. 2003. The effect of prey species and environmental complexity on the functional response of Franklinothrips orizabensis: a test of the fractal foraging model. Ecological Entomology 28:309-318.

Holling, C. S. 1966. The functional response of invertebrate predators to prey density. Memoirs of the Entomological Society of Canada 48:1-86.
Holt, R. D. 1977. Predation, apparent competition, and the structure of prey communities. Theoretical Population Biology 12:197-229.

Holt, R. D., and B. P. Kotler. 1987. Short-term apparent competition. American Naturalist 130:412-430.

Holt, R. D., and J. H. Lawton. 1994. The ecological consequences of shared natural enemies. Annual Review of Ecology and Systematics 25:495-520.

Kondoh, M. 2003. Foraging adaptation and the relationship between food-web complexity and stability. Science 299: $1388-1391$.

Laska, M. S., and J. T. Wootton. 1998. Theoretical concepts and empirical approaches to measuring interaction strength. Ecology 79:461-476.

Mattila, J., and E. Bonsdorff. 1998. Predation by juvenile flounder (Platichthys flesus L.): a test of prey vulnerability, predator preference, switching behaviour and functional response. Journal of Experimental Marine Biology and Ecology 227:221-236.

Müller, C. B., and H. C. J. Godfray. 1999. Indirect interactions in aphid-parasitoid communities. Researches on Population Ecology 41:93-106.

Murdoch, W. W. 1969. Switching in general predators: experiments on predator specificity and stability of prey populations. Ecological Monographs 39:335-354.

Murdoch, W. W. 1973. The functional response of predators. Journal of Applied Ecology 10:335-342.

Murdoch, W. W., S. Avery, and M. E. B. Smyth. 1975. Switching in predatory fish. Ecology 56:1094-1105.

Murdoch, W. W., and A. Oaten. 1975. Predation and population stability. Advances in Ecological Research 9:2131.

Real, L. A. 1977. Kinetics of functional response. American Naturalist 111:289-300.

Schenk, D., and S. Bacher. 2002. Functional response of a generalist insect predator to one of its prey species in the field. Journal of Animal Ecology 71:524-531.

Schenk, D., L. F. Bersier, and S. Bacher. 2005. An experimental test of the nature of predation: neither prey- nor ratiodependent. Journal of Animal Ecology 74:86-91.

Strauss, S. Y. 1991. Indirect effects in community ecology: their definition, study and importance. Trends in Ecology and Evolution 6:206-210.

Tschanz, B., E. Schmid, and S. Bacher. 2005. Host plant exposure determines larval vulnerability-Do prey females know? Functional Ecology 19:391-395.

Wootton, J. T. 1994. The nature and consequences of indirect effects in ecological communities. Annual Review of Ecology and Systematics 25:443-466.

Wootton, J. T. 2002. Indirect effects in complex ecosystems: recent progress and future challenges. Journal of Sea Research 48:157-172.

Witt, R. 1998. Vespidae-Faltenwespen. Pages 168-193 in R. Witt, editor. Wespen beobachten, bestimmen. Naturbuch Verlag, Augsburg, Germany.

Yodzis, P. 2000. Diffuse effects in food webs. Ecology 81:261266. 